\title{
Introdução à cinemática via cálculo de Lagrange: Discutindo os conceitos de velocidades média e instantânea
}

\author{
Introduction to kinematics through Lagrange calculus: Discussing the concepts of average and \\ instantaneous speed
}

\author{
Wagner T. Jardim, Victor J. Vasquez Otoya, José Rogério de Souza
}

Instituto Federal de Educação, Ciência e Tecnologia do Sudeste de Minas Gerais, Juiz de Fora, MG, Brasil

Recebido em 29 de agosto de 2015. Aceito em 24 de outubro de 2015

\begin{abstract}
Este trabalho visa introduzir ideias elementares no estudo da cinemática sob o ponto de vista do cálculo algébrico descrito por Lagrange. Pretendemos demostrar que esta seria uma alternativa ao caminho usualmente adotado no qual se utiliza o cálculo diferencial como base para discutir conceitos como "velocidade instantânea" e "velocidade média".

Palavras-chave: cálculo algébrico de Lagrange, cinemática, plano inclinado de Galileu.
\end{abstract}

This paper aims to introduce basic ideas about kinematics using the algebraic calculus described by Lagrange. We show that this approach is an alternative way to the usually adopted approach that uses differential calculus as a basis for discussing concepts such instantaneous and average speed.

Keywords: Lagrange's algebraic calculus, kinematics, Galileu's inclined plan.

\section{Introdução}

Em diversos livros de Ciências, deparamo-nos com a falta de precisão na definição de grandezas ou com uma linguagem que desfavorece um entendimento mais aprofundado de alguns conceitos. No caso da Física, conceitos ditos básicos - tais como força, massa, velocidade, aceleração e espaço -, muitas vezes são definidos de forma superficial e apresentados a estudantes e a professores em formação sem um aprofundamento adequado, o que pode comprometer discussões futuras que se referem a temas tanto da Física Clássica, quanto da Física Moderna [1. Tais definições e conceitos, apesar de utilizados de forma recorrente por estudantes, podem carregar dificuldades conceituais subtendidas. Podemos citar, como exemplo, a definição de velocidade, na qual, em sua introdução, seria relevante discutir de forma mais rigorosa, diferenciando-a do conceito de rapidez [2]. Uma das definições utilizadas sem o devido rigor é

${ }^{*}$ Endereço de correspondência: wagner.jardim@ifsudestemg.edu.br. a de velocidade média $\left(v_{m}\right)$ usualmente apresentada por

$$
v_{m}=\frac{\triangle x}{\triangle t}
$$

em que, em um percurso, $\Delta \mathrm{x}$ representa o deslocamento e $\Delta$ t, o intervalo de tempo decorrido. A velocidade média $-v_{m^{-}}$pouco nos permite analisar ou extrair informações relevantes sobre o movimento em si por se tratar da média estatística (temporal) de uma grandeza somada ponto a ponto à qual, comumente, referenciamos como velocidade instantânea $\left(v_{i}\right)$. Esta, por sua vez, geralmente nos é apresentada em livros didáticos como

$$
v_{i}=\lim _{\triangle t \longrightarrow 0} \frac{\triangle x}{\triangle t} .
$$

Essa representação do que chamamos de "velocidade instantânea" carrega consigo possíveis problemas conceituais referentes à própria formulação matemática que lhe embasa: a tentativa de expressar o valor de uma função em um ponto através de uma aproximação (limite). Durante muito tempo, o cálculo formalizado por Newton e Leibniz foi alvo 
de muitas críticas devido à difícil compreensão de conceitos envolvidos nessa formulação, tais como fluxões, infinitésimos e diferenciais [3] 7]. Alguns desses conceitos incitaram muitas discussões ao longo da história, sendo, mesmo antes de Newton e Leibniz, utilizados e questionados [8].

As barreiras conceituais ao se trabalhar com os infinitesimais que estruturam as bases do cálculo refletem-se em diversas discussões acerca do assunto e dos seus obstáculos epistemológicos 9 11. Em particular, nos resultados de cálculos referentes a taxas de variação de uma função (derivadas), existem termos (de segunda ordem) que são desconsiderados de forma automática, entretanto, se analisarmos a fundo, percebemos que o porquê de desprezarmos tais termos não é trivial [12,13. Assim, em uma primeira abordagem do conceito de velocidade a partir do cálculo diferencial, ou negligenciamos a desconsideração de termos matemáticos de ordem superiores sem maiores cuidados (o que geralmente é feito), ou nos deparamos com um formalismo mais detalhado que pode fugir aos objetivos de disciplinas básicas em que a velocidade é discutida inicialmente. O formalismo utilizado no desenvolvimento do cálculo diferencial e problemas relacionados interessaram grandes matemáticos como Euler e Lagrange, o primeiro estruturou o chamado cálculo racional e o segundo, o cálculo algébrico. No presente trabalho, pretendemos mostrar como o formalismo desenvolvido por Lagrange no início do Século XIX [14, 15] pode contribuir como alternativa ou complementação na exploração inicial do conceito de velocidade a partir de estudos como o plano inclinado de Galileu, uma vez que, partindo de uma formulação algébrica, os conceitos envolvidos apresentam-se menos abstratos.

\section{O cálculo algébrico de Lagrange}

Nesta seção, apresentaremos como um tratamento algébrico baseado na obra de Lagrange [14,15] pode se constituir em uma alternativa ao uso do conceito de limite.

Para toda função que pode ser (ao menos localmente) expresada como série de potências, temos:

$$
f(x)=a_{0}+a_{1} x+a_{2} x^{2}+\cdots
$$

avaliando no ponto $x=x_{0}$, temos:

$$
f\left(x_{0}\right)=a_{0}+a_{1} x_{0}+a_{2} x_{0}^{2}+\cdots \quad .
$$

Inserindo um acréscimo $\xi$,

$$
\begin{aligned}
& f\left(x_{0}+\xi\right)=a_{0}+a_{1}\left(x_{0}+\xi\right)+a_{2}\left(x_{0}+\xi\right)^{2}+\cdots \\
& =\left(a_{0}+a_{1} x_{0}+a_{2} x_{0}^{2}+\cdots\right)+ \\
& \left(a_{1}+2 a_{2} x_{0}+3 a_{3} x^{2}+\cdots\right) \xi+ \\
& +\left(a_{2}+3 a_{2} x_{0}+\cdots\right) \xi^{2}
\end{aligned}
$$

tomando $\xi=x-x_{0}$, obtemos:

$$
\begin{aligned}
& f(x)=D_{0} f\left(x_{0}\right)+D_{1} f\left(x_{0}\right)\left(x-x_{0}\right)+ \\
& \frac{1}{2 !} D_{2} f\left(x_{0}\right)\left(x-x_{0}\right)^{2}+\cdots
\end{aligned}
$$

em que temos definido: $D_{0} f\left(x_{0}\right)=f\left(x_{0}\right)=a_{0}+$ $a_{1} x_{0}+a_{2} x_{0}^{2}+\cdots, D_{1} f\left(x_{0}\right)=a_{1}+2 a_{2} x_{0}+3 a_{3} x^{2}+$ $\cdots, D_{2} f\left(x_{0}\right)=2 a_{2}+2 \times 3 a_{2} x_{0}+\cdots$. Esta série é chamada de série de Taylor.

Definamos a quantidade, $T_{x_{0}}[f(x)]=D_{0} f\left(x_{0}\right)+$ $D_{1} f\left(x_{0}\right)\left(x-x_{0}\right)$, esta descreve uma reta tangente à curva $f(x)$ no ponto $x_{0}$, e $D_{1} f\left(x_{0}\right)$ é o coeficiente angular ou tangente, o qual chamaremos de derivada.

Observe que, no caso de $x-x_{0}$ ser uma quantidade muito pequena e pudermos desconsiderar os termos de ordens superiores nessa série, teremos $f(x) \approx T_{x_{0}}[f(x)]=D_{0} f\left(x_{0}\right)+D_{1} f\left(x_{0}\right)\left(x-x_{0}\right)$, neste caso, $f(x) \approx f\left(x_{0}\right)+D_{1} f\left(x_{0}\right)\left(x-x_{0}\right)$, que podemos escrever de forma equivalente como

$$
\begin{array}{r}
D_{1} f\left(x_{0}\right)=\lim _{x \rightarrow x_{0}} \frac{f(x)-f\left(x_{0}\right)}{x-x_{0}}= \\
\lim _{\triangle x \rightarrow 0} \frac{f\left(x_{0}+\triangle x\right)-f\left(x_{0}\right)}{\triangle x}
\end{array}
$$

sendo $\triangle x=x-x_{0}$, recuperamos o conceito de derivada. A vantagem do método anterior é alcançarmos os resultados precisos sem a necessidade do conceito formal de limite.

É facil ver, ainda, que para o caso particular $f(x)=x^{\mathrm{n}}$, para $n \in \mathbb{N}, D_{1} f(x)=n x^{n-1}, D_{2} f(x)=$ $n(n-1) x^{n-2}, \quad D_{3} f(x)=n(n-1)(n-2) x^{n-3}$, etc.

Observemos como é simples obter, como exemplo, o que conhecemos como derivada do produto de duas funções. Sejam $f(x)=a_{0}+a_{1} x+a_{2} x^{2}+\cdots$ e $g(x)=b_{0}+b_{1} x+b_{2} x^{2}+\cdots, \operatorname{logo}$ 


$$
\begin{gathered}
T_{x_{0}}^{1}[f(x) g(x)]= \\
{\left[D_{0} f\left(x_{0}\right)+D_{1} f\left(x_{0}\right)\left(x-x_{0}\right)\right]} \\
{\left[D_{0} g\left(x_{0}\right)+D_{1} g\left(x_{0}\right)\left(x-x_{0}\right)\right]=} \\
D_{0} f\left(x_{0}\right) D_{0} g\left(x_{0}\right)+\left[D_{1} f\left(x_{0}\right) D_{0} g\left(x_{0}\right)+\right. \\
\left.D_{0} f\left(x_{0}\right) D_{1} g\left(x_{0}\right)\right]\left(x-x_{0}\right)= \\
f\left(x_{0}\right) g\left(x_{0}\right)+\left[D_{1} f\left(x_{0}\right) g\left(x_{0}\right)+\right. \\
\left.f\left(x_{0}\right) D_{1} g\left(x_{0}\right)\right]\left(x-x_{0}\right)
\end{gathered}
$$

por outro lado

$$
\begin{aligned}
& T_{x_{0}}^{1}[f(x) g(x)]=D_{0}\left[f\left(x_{0}\right) g\left(x_{0}\right)\right]+ \\
& D_{1}\left[f\left(x_{0}\right) g\left(x_{0}\right)\right]\left(x-x_{0}\right)= \\
& f\left(x_{0}\right) g\left(x_{0}\right)+D_{1}\left[f\left(x_{0}\right) g\left(x_{0}\right)\right]\left(x-x_{0}\right)
\end{aligned}
$$

assim,

$$
\begin{gathered}
D_{1}\left[f\left(x_{0}\right) g\left(x_{0}\right)\right]= \\
D_{1} f\left(x_{0}\right) g\left(x_{0}\right)+f\left(x_{0}\right) D_{1} g\left(x_{0}\right)
\end{gathered}
$$

que é a chamada regra de Leibniz ou regra do produto (regra fundamental que define uma derivação).

Lagrange 14, 15 demonstra que este procedimento pode ser realizado com diferentes tipos de funções, tais como racionais, exponênciais, logaritmicas, trigonométricas, etc: 1

\section{Cinemática via Lagrange e o Plano In- clinado de Galileu}

$\mathrm{Na}$ introdução de conceitos relacionados à Cinemática no Ensino, podemos destacar a queda dos corpos tratada por Galileu e a inserção da análise do plano inclinado em sua obra Duas novas ciências [16]. Essa análise é refenciada em diversos trabalhos que indicam discussões acerca dos estudos desenvolvidos por Galileu Galilei como caminho viável no ensino da Física, seja sob um enfoque histórico 17, 18 ou experimental 19 21]. Galileu observou que o módulo da velocidade de um corpo ao descer por um plano inclinado aumenta à medida em que descreve sua trajetória descendente, percebendo a relação entre a distância percorrida e o tempo em um movimento uniformemente acelerado. Galileu demonstrou que o gráfico da distância pelo tempo

\footnotetext{
${ }^{1}$ Apresentaremos alguns exemplos adicionais nos Apêndices do presente artigo.
}

constitui uma parábola, observando, ainda, que, ao subir o plano inclinado, a velocidade diminui até chegar a zero e a relação distância por tempo continua a descrever uma parábola. A diferença entre os gráficos nas funções que representam os dois casos considerados é a inclinação das curvas que varia ponto a ponto. No primeiro caso (descida), a inclinação aumenta e no segundo caso (subida), a inclinação da curva diminui ponto a ponto. Galileu também observou que a velocidade poderia se manter constante (desde que não sofresse influências externas), uma das grandes contribuições à lei da inércia. Mostraremos a seguir que utilizar a formulação algébrica de Lagrange consiste em um caminho conceitualmente simples de se obter as equações básicas do movimento. No último caso mencionado (velocidade constante), o grafico "distância percorrida em função do tempo" é representado por uma linha reta, tendo sua inclinação mantida constante para todo instante de tempo. A equação que descreve esta linha reta é dada por

$$
x(t)=x_{0}+v t
$$

em que $x_{0}=x(0)$ e $v$ é a inclinação da reta. Como a inclinação não varia, é fácil ver que outra forma de obtermos a descrição para a mesma inclinação é tomando dois pontos quaisquer $x_{1}$ e $x_{2}$, nos instantes $t_{1}$ e $t_{2}$, com isto $\frac{\Delta x}{\Delta t}=v$, em que $\triangle x=x_{2}-x_{1}$, e $\triangle t=t_{2-} t_{1}$.

Assim, definimos a velocidade em um ponto como a inclinação da curva neste ponto.Vemos que, no caso particular do movimento retilíneo, podemos obter a velocidade como a distância percorrida dividida pelo tempo, mas isso nao é verdade no caso geral. Vejamos, por exemplo, o caso do plano inclinado. Seja a parábola

$$
x(t)=A+B t+C t^{2}
$$

para calcular a velocidade no instante $t_{0}$, tomemos $x\left(t_{0}\right)$,

$$
x\left(t_{0}\right)=A+B t_{0}+C t_{0}^{2}
$$

logo, tomemos a posição para tempos $t_{0}+\tau$,

$$
\begin{aligned}
& x\left(t_{0}+\tau\right)=A+B\left(t_{0}+\tau\right)+C\left(t_{0}+\tau\right)^{2} \\
& =\left(A+B t_{0}+C t_{0}^{2}\right)+\left(B+2 C t_{0}\right) \tau+C \tau^{2}
\end{aligned}
$$

para $\tau=t-t_{0}$ 


$$
\begin{gathered}
x(t)=\left(A+B t_{0}+C t_{0}^{2}\right)+ \\
\left(B+2 C t_{0}\right)\left(t-t_{0}\right)+C\left(t-t_{0}\right)^{2}
\end{gathered}
$$

para encontrar a velocidade no instante $t_{0}$, tomemos a aproximação linear, ou seja, a reta tangente que passa pelo ponto no instante $t_{0}$,

$$
\begin{gathered}
T_{t_{0}}[x(t)]=\left(A+B t_{0}+C t_{0}^{2}\right)+ \\
\left(B+2 C t_{0}\right)\left(t-t_{0}\right)
\end{gathered}
$$

logo, obtemos a velocidade neste ponto como sendo a inclinação desta reta, ou seja,

$$
v\left(t_{0}\right)=B+2 C t_{0}
$$

para qualquer instante arbitrário

$$
v(t)=B+(2 C) t
$$

observe que, neste caso, o gráfico da velocidade por tempo descreve uma linha reta com interseção em $B=v(0)=v_{0}$ e inclinação $a=2 C$. A inclinação desta reta é chamada aceleração que, no caso particular (aceleração constante), pode ser encontrada da mesma forma que no movimento com velocidade uniforme, tomando dois pontos quaisquer, $\operatorname{logo} a=\frac{\Delta v}{\triangle t}$.

Finalmente, substituindo os resultados,

$$
\begin{gathered}
x(t)=x_{0}+v_{0} t+\frac{a}{2} t^{2} \\
v(t)=v_{0}+a t
\end{gathered}
$$

Podemos realizar o procedimento inverso, isto é: dado o campo de velocidades $v(t)=v_{0}+a t$, qual é a forma da trajetória em função do tempo?

Desde que a trajetória possa ser expressa por uma série de Taylor ao redor do ponto $t=t_{0}$, temos

$$
\begin{gathered}
x(t)=x\left(t_{0}\right)+\dot{x}\left(t_{0}\right)\left(t-t_{0}\right)+ \\
\frac{1}{2 !} \ddot{x}\left(t_{0}\right)\left(t-t_{0}\right)^{2}+\frac{1}{3 !} \dddot{x}\left(t_{0}\right)\left(t-t_{0}\right)^{3}+\cdots
\end{gathered}
$$

em $t=0$

$$
\begin{aligned}
& x(0)=x\left(t_{0}\right)+\dot{x}\left(t_{0}\right)\left(-t_{0}\right)+ \\
& \frac{1}{2 !} \ddot{x}\left(t_{0}\right)\left(-t_{0}\right)^{2}+\frac{1}{3 !} \dddot{x}\left(t_{0}\right)\left(-t_{0}\right)^{3}+\cdots
\end{aligned}
$$

$\log$,

$$
\begin{aligned}
& x\left(t_{0}\right)=x(0)+\dot{x}\left(t_{0}\right) t_{0}- \\
& \frac{1}{2 !} \ddot{x}\left(t_{0}\right) t_{0}^{2}+\frac{1}{3 !} \dddot{x}\left(t_{0}\right) t_{0}^{3}+\cdots
\end{aligned}
$$

substituindo $\dot{x}\left(t_{0}\right)=v_{0}+a t_{0}$ e $\ddot{x}(t)=a, \dddot{x}=\dddot{x}=$ $\cdots=0$, logo para $t_{0}$ arbitrário,

$$
\begin{aligned}
x(t) & =x(0)+\left(v_{0}+a t\right)(t)-\frac{1}{2 !} a(t)^{2} \\
& =x_{0}+v_{0} t+\frac{a}{2} t^{2}
\end{aligned}
$$

reobtendo a expressão conhecida como a descrição da trajetória de uma partícula sob aceleração constante sem a necessidade do processo de cálculo integral.

\section{Resultados e conclusões}

Discutimos no presente trabalho uma forma alternativa de abordar conceitos envolvidos no estudo da Cinemática através da formulação algébrica proposta por Lagrange. Apesar de encontramos na literatura relevantes trabalhos que apontem a obra de Galileu, e em específico o estudo do plano inclinado como um interessante background na análise do movimento acelerado como prática de ensino, muitas vezes, conceitos básicos envolvidos - como o de velocidade - são apresentados sem a devida atenção. Ao introduzir conceitos como o de velocidade instântanea através do cálculo de limite, partimos da defininção de velocidade média em que fazemos o valor de $t$ tender a $t_{0}$, obtendo um resultado aproximado que pode carregar algumas implicações conceituais. O método algébrico de Lagrange se mostra uma interessante alternativa, pois:

1. Não precisamos nos ater à definição de limite, podendo utilizar um formalismo alternativo que leva a resultados finais matematicamente equivalentes aos do cálculo diferencial.

2. Traz estruturas matemáticas mais ricas, tais como a Série de Taylor e a regra de Leibniz (estrutura de derivação).

Pretendemos, assim, resgatar o formalismo algébrico de Lagrange apresentando uma possibilidade alternativa (ou complementar) de abordagem que não necessite de se apoiar na definição de velocidade média nem na formulação baseada no estudo de limites. Além disso, com base nos argumentos desenvolvidos ao longo do presente trabalho, podemos 
concluir que a utilização do termo instantânea como acréscimo à velocidade se mostra redundante, uma vez que velocidade já supõe algo definido em um ponto, o que pode se apresentar confuso ao citarmos diferentes conceitos de velocidade sem a devida atenção.

\section{Apêndice}

Apresentaremos aqui alguns casos particulares importantes a partir do formalismo Algébrico de Lagrange.

\section{Funções racionais}

Seja por exemplo $f(x)=x^{p}$, para $p$ racional, logo

$$
f\left(x_{0}+\xi\right)=\left(x_{0}+\xi\right)^{p}=x_{0}(1+\delta)^{p}
$$

em que $\delta=\frac{\xi}{x_{0}}$. Observe que $(1+\delta)^{p}=1+F(p) \delta+$ $\cdots$. em que $F(p)$ é uma função arbitrária. Vejamos o produto,

$$
\begin{array}{r}
(1+\delta)^{p}(1+\delta)^{q}=(1+F(p) \delta+\cdots) \\
(1+F(q) \delta+\cdots)=1+(F(p)+F(q)) \delta+\cdots
\end{array}
$$

por outro lado,

$$
\begin{aligned}
& (1+\delta)^{p}(1+\delta)^{q}= \\
& (1+\delta)^{p+q}=1+F(p+q) \delta+\cdots
\end{aligned}
$$

assim temos

$$
F(p+q)=F(p)+F(q) .
$$

Usando esta última identidade obtemos

$$
F(p)+F(q)=F(p+r)+F(q-r)
$$

desenvolvendo em série deTaylor

$$
\begin{gathered}
F(p+r)= \\
D_{0} F(p)+D_{1} F(p) r+\frac{1}{2} D_{2} F(p) r+\cdots \\
F(q-r)= \\
D_{0} F(q)-D_{1} F(q) r+\frac{1}{2} D_{2} F(q) r+\cdots
\end{gathered}
$$

substituindo, temos que $D_{1} F(p)-D_{1} F(q)=0$, $D_{2} F(p)+D_{2} F(q)=0, \ldots$, logo escolhemos consistentemente $D_{1} F(p)=D_{1} F(q)=a, D_{2} F(p)=$ $D_{2} F(q)=0, \ldots$. . Com isto,

$$
F(p+r)=F(p)+a r
$$

escolhendo $r=-p$, temos $F(0)=F(p)-a p$, ou seja, $F(p)=F(0)+a p$. Voltando para

$$
\begin{aligned}
(1+\delta)^{p} & =1+F(p) \delta+\cdots \\
& =1+[F(0)+a p] \delta+\cdots
\end{aligned}
$$

para $p=0$, vemos imediatamente que $F(0)=0$; para $p=1$, vemos que $a=1$. Concluimos que para $p$ arbitrario $(1+\delta)^{p}=1+p \delta+\cdots$

$$
\begin{aligned}
f\left(x_{0}+\xi\right) & =\left(x_{0}+\xi\right)^{p}= \\
x_{0}^{p}(1+\delta)^{p} & =x_{0}^{p}+p x_{0}^{p-1} \xi+\cdots
\end{aligned}
$$

em $\xi=x-x_{0}$, assim

$$
\begin{gathered}
T_{x_{0}} f(x)=x_{0}^{p}+p x_{0}^{p-1}\left(x-x_{0}\right)+\cdots \\
D_{1} f\left(x_{0}\right)=D_{1}\left(x^{p}\right)=p x_{0}^{p-1}
\end{gathered}
$$

$\operatorname{logo}, D_{2}\left(x_{0}^{p}\right)=p(p-1) x_{0}^{p-2}, D_{3}\left(x_{0}^{p}\right)=$ $p(p-1)(p-2) x_{0}^{p-3} \ldots$ o que nos leva a

$$
\begin{aligned}
& (x+\xi)^{p}=x^{p}+p x^{p-1} \xi+\frac{1}{2 !} p(p-1) x^{p-2} \xi^{2}+ \\
& \frac{1}{3 !} p(p-1)(p-2) x^{p-3} \xi^{3}+\cdots
\end{aligned}
$$

\section{Funções exponenciais e logarítimicas}

Outro exemplo importante é a função $f(x)=a^{x}$,

$$
f(x+\xi)=a^{x+\xi}=a^{x} a^{\xi}
$$

seja $a=1+b$, logo,

$$
\begin{aligned}
& (1+b)^{\xi}=1+\xi b+\frac{1}{2 !} \xi(\xi-1) b^{2}+ \\
& \frac{1}{3 !} \xi(\xi-1)(\xi-2) b^{3}+\cdots= \\
& 1+\xi\left[b-\frac{1}{2} b^{2}+\frac{1}{3} b^{3}+\cdots\right]+\cdots
\end{aligned}
$$




$$
\begin{aligned}
& a^{\xi}=1+\xi\left[(a-1)-\frac{1}{2}(a-1)^{2}+\right. \\
& \left.\frac{1}{3}(a-1)^{3}+\cdots\right]+\cdots
\end{aligned}
$$

escrevemos $a^{\xi}=1+c \xi+\cdots$, em que $c=(a-1)-$ $\frac{1}{2}(a-1)^{2}+\frac{1}{3}(a-1)^{3}+\cdots, \operatorname{logo}$

$$
f(x+\xi)=a^{x+\xi}=a^{x}+c a^{x} \xi+\cdots
$$

para $\xi=x-x_{0}$

$$
T_{x_{0}} f(x)=a^{x}+c a^{x}\left(x-x_{9}\right)
$$

assim temos que $D_{1} f=c a^{x}, \operatorname{logo} D_{2} f=c^{2} a^{x}$, $D_{3} f=c^{3} a^{x}$. o que nos leva a

$$
a^{x+\xi}=a^{x}+c a^{x} \xi+\frac{1}{2 !} c^{2} a^{x} \xi^{2}+\cdots
$$

para $x=0, \log \mathrm{o}$

$$
a^{\xi}=1+c \xi+\frac{1}{2 !} c^{2} \xi^{2}+\frac{1}{3 !} c^{3} \xi^{3}+\cdots
$$

para $\xi=1$, o número $a$ pode ser escrito como a série

$$
a=1+c+\frac{1}{2 !} c^{2}+\frac{1}{3 !} c^{3}+\cdots
$$

para $c=1$,

$$
e=1+1+\frac{1}{2 !}+\frac{1}{3 !}+\frac{1}{4 !}+\cdots
$$

assim,

$$
e^{\xi}=1+\xi+\frac{1}{2 !} \xi^{2}+\frac{1}{3 !} \xi^{3}+\cdots
$$

Voltemos a $a^{\xi}$ e escolhemos $\xi=\frac{1}{c}$, temos

$$
a^{1 / c}=1+1+\frac{1}{2 !}+\frac{1}{3 !}+\cdots=e
$$

$\log$,

$$
\begin{gathered}
a=e^{c} \Longrightarrow c=\log _{e} a \equiv \ln a \\
a^{x+\xi}=a^{x}+\ln a a^{x} \xi+\frac{1}{2 !}(\ln a)^{2} a^{x} \xi^{2}+\cdots
\end{gathered}
$$

assim, obtém-se

$$
D_{1} a^{x}=\ln a a^{x}
$$

$$
\ln a=(a-1)-\frac{1}{2}(a-1)^{2}+\frac{1}{3}(a-1)^{3}+\cdots .
$$

Da última equação, temos

$$
\begin{aligned}
& \ln (x+\xi)=(x+\xi-1)-\frac{1}{2}(x+\xi-1)^{2}+ \\
& \frac{1}{3}(x+\xi-1)^{3}+\cdots=\left[(x-1)-\frac{1}{2}(x-1)^{2}+\right. \\
& \left.\frac{1}{3}(x-1)^{3}+\cdots\right]+\left(\frac{1}{x}\right) \xi+\cdots
\end{aligned}
$$

usando o resultado obtido anteriormente para funções racioanais

$$
\begin{aligned}
& \frac{1}{x}=\frac{1}{1+(x-1)}= \\
& 1-(x-1)+(x-1)^{2}-(x-1)^{3}+\cdots
\end{aligned}
$$

$\log \mathrm{O}$

$$
D_{1} \ln x=\frac{1}{x}
$$

\section{Funções trigonométricas}

Para encontrar a derivada das funções trigonométricas, partimos da fórmula de euler

$$
e^{i \theta}=\cos \theta+i \sin \theta .
$$

É facil identificar as funções $\sin \theta$ e $\cos \theta, \operatorname{com}$ a expansão da exponencial

$$
\begin{aligned}
& \cos \theta=1-\frac{\theta^{2}}{2 !}+\frac{\theta^{4}}{4 !}-\frac{\theta^{6}}{6 !}+\cdots \\
& \sin \theta=\theta-\frac{\theta^{3}}{3 !}+\frac{\theta^{5}}{5 !}-\frac{\theta^{7}}{7 !}+\cdots
\end{aligned}
$$

usando $D_{1} f(x)=n x^{n-1}$, vemos que

$$
D_{1}[\cos \theta]=-\sin \theta \quad \text { e } \quad D_{1}[\sin \theta]=\cos \theta
$$

\section{Função implícita (Regra da cadeia)}

Seja $f=f(g(x))$, logo

$$
\begin{aligned}
& f(g(x+\xi))= \\
& f\left(g(x)+D_{1} g(x) \xi+D_{2} g(x) \frac{\xi^{2}}{2 !}+\cdots\right)= \\
& f(g(x)+h(\xi))= \\
& f(g(x))+D_{1}^{g} f(x) h(\xi)+\cdots= \\
& f(g(x))+D_{1}^{g} g(x) D_{1} g(x) \xi+\cdots
\end{aligned}
$$


vemos então que

$$
\begin{array}{r}
T^{1}[f(g(x+\xi))]= \\
f(g(x))+D_{1}^{g} g(x) D_{1} g(x) \xi
\end{array}
$$

com

$$
D_{1}[f(g(x))]=D_{1}^{g} f(x) D_{1} g(x) .
$$

\section{Referências}

[1] W.T. Jardim, V.J.V. Otoya e C.G.S. Oliveira, Revista Brasileira de Ensino de Física, 37, 2506 (2015).

[2] P.M. Mors, Revista Brasileira de Ensino de Física 30, 2101 (2008).

[3] M. B. W. Tent Leonhard Euler and the Bernoullis (AA K Peters/CRC Press, Natick, 2009).

[4] J.V. Garbinet, The Origins of Cauchy's Rigourous Calcullus (The MIT Press, Cambridge, 1981).

[5] V.J. Katz, A History of Mathematics: An Introduction (Harper Collins College Publishers, New York, 1993).

[6] H.J. Keisler, Foundations of Infinitesumal Calculus (Prindle, Weber \& Shmidt. Inc., Boston, 1976).

[7] B. Vermeulen, Berkley Newsletter 8, 1 (1985).

[8] J.M.F. Bassalo, Revista Brasileira de Ensino de Física 18, 103 (1996).

[9] A. Sierpinska, Educational Studies in Mathematics 18, 371 (1987).

[10] D. Tall in: Handbook of Research on Mathematics Teaching and Learning, editado por D. Grouws (Macmillan Publishing Co, New York, 1992), p. 495-511.

[11] I. Zuchi, A Abordagem do Conceito de Limite via Sequência Didática. Tese de Doutorado, Universidade Federal de Santa Catarina, 2005.

[12] K.Marx, Mathematical Manuscripts of Karl Marx (New York Publication Ltd., New York, 1983).

[13] C.I. Castillo Analisis Matematico, disponível em http://www.uv.es/ivorra/Libros/Libros.htm acesso 25/08/2015.

[14] J.L. Lagrange Leçons sur Lel Calcul Des Functions (Impr Libraire pour le Mathématiques, Paris, 1806).

[15] J.L. Lagrange, Théorie des Functions Analytiques (Impr Libraire pour le Mathématiques, Paris, 1813).

[16] A. Zylbersztajn, Caderno Brasileiro de Ensino de Física, 5, 36 (1988).

[17] T. Peron, A. Guerra e T.C. Forato, in: Anais do VIII Encontro Nacional De Pesquisa Em Educação E Ciências, Campinas, 2011, disponível em http://www.nutes.ufrj.br/abrapec/ viiienpec/resumos/R0411-2.pdf.

[18] M.C.D. Neves, J.M. Batista, J.R. Costa, L.C. Gomes. M.C. Batista, P.A. Fusinato. F.R. Almeida, R.G.R. Silva, A.A. Savi y R.F. Pereira, Revista Electrónica de Enseñanza de las Ciencias, 7, 226 (2008).
[19] P. Cerreta, Science \& Education, 23, 747 (2014).

[20] R.R. Soares e P.F. Borges, Revista Brasileira de Ensino de Física, 32, 2501 (2010).

[21] S. Straulino, Physics Education, 43, 316 (2008). 